\title{
Tratamiento crítico de la información de estudiantes universitarios desde los entornos personales de aprendizaje
}

\author{
José Luis Serrano Sánchez ${ }^{1}$ \\ ORCID: 0000-0003-2359-959X \\ Xavier Carrera Farrán ${ }^{2}$ \\ ORCID: 0000-0003-3420-4215 \\ Enric Brescó Baiges ${ }^{2}$ \\ ORCID: 0000-0001-8477-6970 \\ Cristóbal Suárez-Guerrero ${ }^{3}$ \\ ORCID: 0000-0002-6558-4321
}

\section{Resumen}

Partiendo del Proyecto CAPPLE (financiado por el Ministerio español de Economía y Competitividad), en este artículo identificamos las estrategias y las herramientas en red utilizadas por 2054 estudiantes universitarios españoles de último curso para efectuar un tratamiento crítico de la información y gestionar su proceso de aprendizaje. Desde un diseño de investigación no experimental de tipo exploratorio, se ha utilizado un cuestionario validado a través de un triple procedimiento (juicio de expertos, entrevistas cognitivas y estudio piloto) para recoger datos de 75 universidades en España. Los resultados muestran que los futuros profesionales son conscientes de la necesidad de contrastar y aplicar un pensamiento crítico sobre la información que reciben; otorgan credibilidad a la información asociada a su ámbito formativo; cuestionan la recibida del exterior y valoran el uso de herramientas de aprendizaje según la valoración académica que esperan tener. Entre los temas pendientes que abre este trabajo se destaca con nitidez la profundización teórica y práctica sobre la capacidad predictiva de los Entornos Personales de Aprendizaje (PLE): a) como herramienta de evaluación y de aprendizaje en futuras acciones de desempeño laboral y; b) en cómo mejora este desempeño tras una sólida formación en el tratamiento crítico de la información desplegada durante los estudios universitarios de grado.

\section{Palabras clave}

Pensamiento crítico - Acceso a la información - Educación superior - Gestión del conocimiento - PLE.

1- Universidad de Murcia, Murcia, España. Contacto: jl.serranosanchez@um.es.

2- Universidad de Lleida, Lleida, España. Contacto: carrera@pip.udl.cat; enric@pip.udl.cat.

3- Universitat de València, Valencia, España. Contacto: cristobal.suarez@uv.es.

(cc) ( \$ DOl: http://dx.doi.org/10.1590/S1678-4634201945193355

(c) BY This content is licensed under a Creative Commons attribution-type BY-NC. 


\title{
Critical processing of information on university students from personal learning environments
}

\begin{abstract}
Based on the CAPPLE Project (funded by the Spanish Ministry of Economy and Competitiveness), in this article we identify the strategies and network tools used by 2054 Spanish university senior students to critically process information and manage their learning process. From a non-experimental research design of exploratory type, a validated questionnaire was used through a triple procedure (expert judgment, cognitive interviews and pilot study) to collect data from 75 Spanish universities. The results show that future professionals are aware of the need to contrast and apply critical thinking about the information they receive; grant credibility to the information associated with their training environment; they question the received from external and value the use of learning tools according to the academic assessment they expect to have. Among the pending issues that this work opens is the theoretical and practical in-depth look at the predictive capacity of Personal Learning Environments (PLE): a) as a tool for evaluation and learning in future work performance actions; b) how this performance improves after a solid formation in the critical processing of the information displayed along the undergraduate studies.
\end{abstract}

\section{Keywords}

Critical thinking - Access to information - Higher education - Knowledge management - PLE.

\section{Pensamiento crítico y Personal Learning Environment (PLE)}

Nos encontramos en un período de cambio en los procesos de enseñanza/aprendizaje que se dan en la formación universitaria, en sintonía con los que también se dan en las etapas educativas previas. No sólo se trata de modificar los procedimientos docentes adaptándolos a los requerimientos competenciales, sino que el cambio de rol de los docentes viene asociado a un mayor protagonismo de los estudiantes en la gestión de su conocimiento (GROS, 2015). Cada vez más el alumnado toma las riendas de su proceso de aprendizaje convirtiéndose en un agente activo e implicado a nivel personal, capaz de reflexionar y de tomar decisiones en la planificación y desarrollo de su formación (LOVELESS; WILLIAMSON, 2017). Este panorama exige al docente dejar de lado prácticas basadas en la reproducción mecánica de la información como motor del aprendizaje y como evidencia en la evaluación.

Ante estas nuevas exigencias educativas sigue estando vigente el desarrollo del pensamiento crítico en los términos en que lo planteaba Lipman (1991). La formación de alumnos 
críticos conlleva trabajar habilidades de pensamiento crítico a pesar de que no resulta fácil conceptualizar el término y mucho menos evaluarlo (DANIEL et al., 2003). Díaz Barriga (2001) recopila distintos enfoques que subyacen en las definiciones del pensamiento crítico, basándose en los trabajos de autores como McMillan (1987), Mayer y Goodchild (1990), Halpern y Nummedal (1995), Monroy (1998), Horn (2000) y Pacheco (2001). En estos estudios se valora el pensamiento crítico de diferentes formas y niveles, en función de la postura y perspectiva de la cual se parta. Por ejemplo, se considera la capacidad de manifestar una opinión personal, un punto de vista más o menos fundamentado, la emisión de un juicio, como parte de un proceso cognitivo complejo en el cual se deben contemplar diferentes habilidades de pensamiento, entre otras. Los autores consideran que el pensamiento crítico también conlleva que los estudiantes tengan unos conocimientos, unas habilidades analíticas, una capacidad para comprender y procesar la información, junto con una actitud reflexiva.

Horn (2000), frente a la dificultad de promover el pensamiento crítico en el aula, plantea una enseñanza basada en el diálogo y la alfabetización crítica, que vaya más allá de una suma de habilidades. La mayoría de los autores coincide con la necesidad de modificar los procesos de aprendizaje tradicionales para favorecer las habilidades de pensamiento.

No hay duda en que los conocimientos previos y el aprendizaje de nuevos fundamentan la reflexión y la toma de decisiones del individuo. Aunque esto no conlleva directamente el desarrollo de competencias cognitivas ni competencias metacognitivas que, según Nieto y Saiz (2011), son básicas para el desarrollo del pensamiento crítico en los alumnos afectando directamente en el proceso de enseñanza/aprendizaje.

La importancia que tiene el pensamiento crítico en la toma de decisiones a las que se enfrentan las personas ya ha sido destacada como sustancial para la vida (BUTTER et al., 2012) y es, en la actualidad, una tendencia cada vez más consistente que describe una línea de investigación multidisciplinar (DWYER; HOGAN; STEWART, 2014). No obstante, en tiempos de sobreabundancia de información en Internet la necesidad de atender de forma crítica la información adquiere otro matiz cuando el entorno de acción y acceso a la información es la red. Este matiz viene dado no sólo por el volumen de datos al que se accede gracias a Internet, que ya por si solo justificaría un punto de inflexión, sino también porque la relación entre el sujeto y la información dadas las nuevas condiciones, flujos y herramientas ha cambiado, permitiendo además del consumo, la creación y la comunicación de información en red (CASTELLS, 2009; DEZUANNI; MONROY, 2012).

Una parte de la necesidad por entender y formar a los ciudadanos de manera crítica sobre la información en red se viene trabajando bajo el paraguas conceptual de competencia digital (CARRETERO; VUORIKARI; PUNIE, 2017; VUORIKARI et al., 2016), que en la versión propuesta por la Unión Europea remite específicamente a dos dimensiones: en los procesos de evaluación de la información y en la solución de problemas (FERRARI, 2013). En este punto existe un esfuerzo cada vez más amplio por estimular la formación en capacidades que vayan más allá del ejercicio técnico en la educación (ALA-MUTKA; PUNIE; REDECKER, 2008; GISBERT; ESTEVE, 2011; PRENDES, 2010). A nivel profesional también se exige la capacidad crítica como un aspecto relacionado con los procesos de curación de contenidos (GUALLAR; LEIVA-AGUILERA, 2013). 
Pero cuando se traslada la necesidad de entender la crítica como parte ineludible en la gestión del potencial de Internet en la construcción de entornos personales de aprendizaje (CONOLE, 2013; KOP, 2011), la exigencia se hace más concreta y, si cabe, transversal a la competencia digital. Esto es, no basta con acceder a la información, sino que la única forma de entender el provecho del acceso al potencial informacional en red en la formación es anteponiendo la capacidad crítica como filtro relevante.

Tanto el entorno como la comunicación en red describen otras condiciones de aprendizaje (SUÁREZ-GUERRERO, 2013) que, a su vez, abren nuevas rutas de acción y representación al conocimiento (BENKLER, 2014). Por ello, la apropiación significativa de las herramientas en red para el aprendizaje pasa no solo por el desarrollo de la capacidad técnica del uso, sino por cuestionar la información a la que se accede, por valorar la credibilidad de las fuentes, por reflexionar sobre la información que se aprende y, en general, por demostrar una actitud crítica ante la información que se procesa. Estos filtros críticos forman parte de este estudio sobre gestión del aprendizaje como parte del desarrollo del Personal Learning Environment (PLE).

Los PLE, como estrategia de representación en un ecosistema educativo en red, es una buena metáfora (WILSON, 1995) para entender la complejidad de los procesos de gestión del aprendizaje en Internet. En este marco, la crítica no es un componente accesorio en la representación y actividad en un PLE y, bien visto, puede ser el valor añadido en el marco de la Educación Superior donde las necesidades de formación son cada vez más amplias y se asocian a actividades ligadas al aprendizaje permanente (JISC, 2012). La gestión del aprendizaje que implica desarrollar un PLE es una buena herramienta -e indicador- potencial de aprendizaje.

Por tanto, cuando los entornos educativos en la formación exigen un desenvolvimiento crítico durante toda la vida y los entornos educativos clásicos, la educación formal, no formal e informal se solapan en la red (KERHOHAN, 2013), hace falta ir más allá de las modas. La percepción del aprendizaje y gestión crítica de la información deben ser vistas como una necesidad formativa que está más allá de la etnicidad digital, asociada a la fecha de nacimiento, como se señalaba en la cuestionable noción de Nativo Digital (PRENSKY, 2001). Ya sea como residentes o visitantes digitales (WHITE; LE CORNU, 2011) -o como generación Y, milenials, net-agers, digital learners, gamer generation, digital wisdom, digitizen, app generation o cyberkid entre otros (GALLARD0, 2015)- el filtro mediacional entre el sujeto y la información de Internet, puede ser el ejercicio crítico de un PLE. Esto también forma parte de la disrupción pedagógica con Internet (Conole et al., 2008).

A pesar de entender el PLE como una buena herramienta para el cambio de los procesos educativos, no hay que olvidar que la construcción consciente del propio PLE requiere de habilidades contrastadas de pensamiento complejo, entre ellas las propias del pensamiento crítico. Por este mismo motivo, en el aula se debe promover y desplegar estrategias de trabajo que puedan aportar una mejora de la implicación de los alumnos en sus propios procesos cognitivos. En otras palabras, es determinante el papel que juega el estudiante en la construcción de su propia identidad, ya que ésta no viene predefinida, sino que evoluciona a lo largo de su vida en función de los roles que se vayan adquiriendo y las decisiones que van tomando, considerando los propios polos identitarios dados históricamente (NAVARRETE-CAZALES, 2015). 
En ese sentido, como señala Burbules (2014), tener una idea de las consecuencias del porqué y cómo organizar la información en entornos complejos, sobreabundantes y globales como son los entornos digitales en red, es de suma importancia tanto para quien aprende, como para quien busca ese desarrollo. No bastan las herramientas de refinamiento de información, hace falta añadir componentes simbólicos de representación Peña-López (2016). Por ello, la construcción de PLE es tanto indicador como promotor de ese desarrollo que es necesario tomar en cuenta en los procesos de aprendizaje.

Considerando la importancia del PLE para la mejora del proceso de aprendizaje de los alumnos, presentamos en este artículo un estudio que toma como punto de partida el proyecto CAPPLE: Competencias para el Aprendizaje Permanente basado en el uso de PLE. Análisis de los futuros profesionales y Propuestas de mejora (Referencia EDU201233256). Proyecto financiado por el Ministerio español de Economía y Competitividad que parte de la necesidad de formar a los futuros profesionales para el uso de herramientas telemáticas y estrategias de aprendizaje que les permitan crear y aprovechar las mejores oportunidades de desarrollo profesional durante el resto de sus vidas (PRENDES, 2013).

\section{Objetivo}

El Proyecto CAPPLE pretende describir y analizar los PLE de los futuros profesionales españoles. La finalidad principal del proyecto es conocer cómo son dichos entornos, las estrategias utilizadas para su configuración y las carencias de formación transversal que presentan. Una parte del proyecto CAPPLE se focaliza en el tratamiento crítico que los estudiantes universitarios realizan de la información que reciben y en el papel que tienen las herramientas en red para apoyar sus aprendizajes, como respuesta a las siguientes preguntas de investigación: ¿Qué determina la variedad y cantidad de herramientas en red que utilizan los estudiantes en su proceso de aprendizaje? ¿Consideran creíble, fiable la información que reciben? ¿La cuestionan? ¿Cómo actúan ante la información que reciben? ¿Utilizan también las herramientas en red para plasmar sus reflexiones sobre aquello que aprenden?

En relación a estas cuestiones, nos planteamos el siguiente objetivo de investigación que tratamos en este artículo: identificar las estrategias y las herramientas en red utilizadas de forma habitual por estudiantes universitarios de último curso para efectuar un tratamiento crítico de la información y gestionar su proceso de aprendizaje, dentro y fuera de las aulas; especialmente aquellas que tienen lugar en contextos electrónicos. El logro de este objetivo contribuirá a que las instituciones universitarias diseñen e implementen estrategias que permitan a los estudiantes desarrollar el tratamiento crítico de la información como elemento clave en la gestión de sus aprendizajes, en el aula y sobre todo, en contextos virtuales.

\section{Diseño de la investigación}

Esta investigación de corte empírico trata de recoger información de tipo descriptiva, sin establecer comparaciones entre grupos ni manipular variables. Por tanto, se ha realizado 
un diseño de investigación no experimental, de tipo exploratorio basado en el uso del cuestionario como instrumento de recogida de información (ATO; LÓPEZ; BENAVENTE, 2013, PARD0; RUIZ; SAN MARTÍN, 2015).

La investigación se llevó a cabo durante los años 2013-2017, desarrollándose en cinco fases de trabajo (PRENDES et al., 2014): revisión teórica sobre los PLE y sobre estudios desarrollados previamente, diseño y validación del instrumento, recogida de información sobre PLE análisis de los datos y descripción del PLE de los alumnos universitarios españoles participantes.

\section{Participantes}

La población de este estudio se corresponde con el conjunto de estudiantes de último curso de licenciatura o grado de todas las universidades españolas públicas y privadas (81 universidades en las que se imparte docencia), es decir, un total de 298000 estudiantes en el curso 2014-2015 (el 54.9\% mujeres y el 45.1\% hombres). Es, por tanto, una población amplia y geográficamente dispersa, lo cual supuso un acceso a los participantes extremadamente complejo. De esta manera, no todos los elementos poblacionales tuvieron la oportunidad de ser elegidos puesto que se optó por una técnica de difusión a los participantes elegidos de modo intencional. Tras finalizar este proceso, se recogieron durante el curso académico 2015-2016 un total de 4399 encuestas de las que fueron válidas 2054 casos (de 75 universidades diferentes), ya que fueron aquellos que completaron todas las preguntas. Esta muestra participante es amplia pero no representativa, hecho que no permite establecer inferencias ni desarrollar conclusiones que se puedan generalizar al resto de población objeto de estudio.

A pesar de esta limitación la muestra se considera útil por el elevado número de participantes $(n=2054)$, y hace posible la obtención de evidencias consistentes y conclusiones robustas sobre el tratamiento crítico que los estudiantes universitarios españoles hacen de la información que emplean en sus procesos de aprendizaje.

De los estudiantes participantes un $69.67 \%$ corresponde a mujeres y un $30.33 \%$ a hombres. En la tabla 1 se muestra la distribución de la población y de la muestra participante por áreas de conocimiento.

Tabla 1- Población y muestra

\begin{tabular}{|c|c|c|c|c|}
\hline Área de Conocimiento & Estudiantes de último curso & Porcentaje de estudiantes de último curso & Muestra & Muestra (\%) \\
\hline Ciencias Sociales y Jurídicas & 153952 & 52.2 & 879 & 42.79 \\
\hline Ingeniería y Arquitectura & 78265 & 26.4 & 277 & 13.49 \\
\hline Artes y Humanidades & 19966 & 6.8 & 308 & 15 \\
\hline Ciencias de la Salud & 29844 & 10.1 & 352 & 17.14 \\
\hline Ciencias & 16044 & 5.4 & 238 & 11.58 \\
\hline Total & 298071 & 100 & 2054 & 100 \\
\hline
\end{tabular}

Fuente: Elaboración propia. 


\section{Cuestionario CAPPLE: exploración de PLE}

En la investigación se ha utilizado como instrumento de recogida de información el cuestionario. Su diseño se basa en tres modelos teóricos: el concepto de PLE (CASTAÑEDA; ADELL, 2011, 2013), el aprendizaje autorregulado (ANDERSON, 2002; MARTÍN et al., 2007; MIDGLEY et al., 2000; PINTRICH et al., 1991) y la comunicación y las competencias TIC (PRENDES, 2010; PRENDES; GUTIÉRREZ, 2013), a partir de los cuales se organizaron los items del cuestionario en cuatro dimensiones (gestión de la información, gestión del proceso de aprendizaje, autopercepción y comunicación).

El cuestionario fue validado a través de un triple procedimiento. En primer lugar, se hizo una validación de constructo a través de juicio de expertos (ocho profesionales en tres rondas consecutivas) por el método de agregados individuales. Posteriormente, se llevaron a cabo entrevistas cognitivas de sondeo concurrente para obtener evidencias sobre la validez de los ítems formulados en el instrumento. Para ello se tuvo en en cuenta la información aportada por veinticuatro estudiantes (UNIVERSIDADES del País Vasco, Oviedo, Las Palmas de Gran Canaria y Murcia) sobre diez ítems cruciales. Tras este segundo proceso, se rediseñó el cuestionario y realizó una prueba piloto con una muestra productora de datos de 224 estudiantes (muestra invitada de 406 sujetos) de las diferentes universidades participantes en el proyecto. El proceso completo de validación del cuestionario (con todas las versiones) se encuentra ampliamente desarrollado en (PRENDES et al., 2016).

Además de los procedimientos de validación indicados, se realizaron pruebas psicométricas con la intención de conocer la fiabilidad de la escala de medida, concretamente encontramos una fiabilidad de 0.944 en la prueba de Alfa de Cronbach.

El cuestionario utilizado está formado por 48 preguntas, de las cuales cuatro son de caracterización demográfica (sexo, edad, universidad y área de conocimiento), ocho preguntas son de tipo nominal y 35 preguntas responden a una escala de cinco niveles (Siempre/casi siempre; A menudo; A veces; Pocas veces; Casi nunca/nunca) más una opción de No usa/No aplicable. De otro lado, treinta de ellas relativas a frecuencia y 5 al grado de acuerdo o desacuerdo con la afırmación. En Prendes y otros autores (2016) se presenta la versión final del mismo, el cual se administró en red a través de la herramienta Survey Monkey utilizando el correo electrónico como vía principal de difusión.

Para dar respuesta al objetivo de investigación en que se centra este artículo se seleccionaron una serie de preguntas centradas en el tratamiento crítico de la información, que a su vez forman parte de la dimensión gestión del aprendizaje del cuestionario CAPPLE.

Cada una de estas cinco preguntas está asociada a una variable específica de cómo los estudiantes universitarios emplean su PLE en su aprendizaje a partir de procesos de valoración, análisis y reflexión crítica de la información a que tienen acceso. La tabla 3 muestra la relación entre cada cuestión con su variable y la definición de cada una de ellas. 
Tabla 2- Preguntas del cuestionario CAPPLE centradas en el pensamiento crítico e ítems de respuesta

\begin{tabular}{|c|c|}
\hline Pregunta $n^{0} 12$ & $\begin{array}{l}\text { El número y la variedad de herramientas en red que utilizo para aprender dependen de: } \\
\text { La importancia que doy a un aprendizaje } \\
\text { El tiempo que tengo para ese aprendizaje } \\
\text { La valoración académica o profesional que va a tener ese aprendizaje } \\
\text { El impacto que tendrá en mi prestigio en red }\end{array}$ \\
\hline Pregunta $n^{0} 19$ & $\begin{array}{l}\text { Cuestiono la información que recibo de... } \\
\text { Mis profesores } \\
\text { Mis amigos y familiares } \\
\text { Medios de comunicación tradicionales } \\
\text { Medios de comunicación en red } \\
\text { Blogs y páginas web } \\
\text { Twitter } \\
\text { Redes sociales } \\
\text { Foros } \\
\text { Tutoriales } \\
\text { Aplicaciones móviles específicas } \\
\text { Noticias que me llegan al correo } \\
\text { De expertos u otros profesionales del área }\end{array}$ \\
\hline Pregunta $n^{0} 20$ & $\begin{array}{l}\text { ¿Qué añade credibilidad a la información que recibo? } \\
\text { Que me lo recomienden mis colegas, amigos y familiares } \\
\text { Que sea recomendado en las redes sociales } \\
\text { Que aparezca en un sistema de recomendación en red (Meneame, Tripavisor) } \\
\text { Que sea una de las primeras posiciones de la búsqueda en Google } \\
\text { Que sea trending topic en Twitter } \\
\text { Que aparezca en varios recursos (artículos, libros, vídeos) en red } \\
\text { Que lo recomiende un experto }\end{array}$ \\
\hline Pregunta n²1 & $\begin{array}{l}\text { Ante la información que recibo: } \\
\text { Soy consciente de que la interpreto según mis propios puntos de vista } \\
\text { La interpreto de manera reflexiva en base a argumentos que me ayuden a comprenderla } \\
\text { Valoro la opinión dada por el grupo de usuarios } \\
\text { Pienso que no siempre es veraz o se corresponde con la realidad } \\
\text { Considero que no debo cuestionarla, sea cual sea su origen } \\
\text { La contrasto }\end{array}$ \\
\hline Pregunta $n^{0} 36$ & $\begin{array}{l}\text { Suelo reflejar la reflexión sobre lo que voy aprendiendo... } \\
\text { En un blog personal } \\
\text { En un documento Word } \\
\text { En mi calendario electrónico } \\
\text { En una hoja de papel o mi diario }\end{array}$ \\
\hline
\end{tabular}

Fuente: Elaboración propia. 
Tabla 3- Relación de variables y correspondencia con las preguntas del cuestionario

\begin{tabular}{|c|c|c|}
\hline Pregunta & Variable & Definición de la variable \\
\hline Pregunta $n^{0} 12$ & $\begin{array}{l}\text { Valoración de herramientas de } \\
\text { aprendizaje }\end{array}$ & $\begin{array}{l}\text { Motivos que contempla el estudiante para la selección de herramientas en red } \\
\text { que utiliza en su aprendizaje. }\end{array}$ \\
\hline Pregunta $n^{0} 19$ & $\begin{array}{l}\text { Credibilidad de la información: según } \\
\text { origen }\end{array}$ & $\begin{array}{l}\text { Grado de veracidad y certeza que el estudiante asigna a la información a que } \\
\text { tiene acceso, según la fuente de procedencia. }\end{array}$ \\
\hline Pregunta $n^{0} 20$ & $\begin{array}{l}\text { Credibilidad de la información: según } \\
\text { recomendación }\end{array}$ & $\begin{array}{l}\text { Grado de veracidad y certeza que el estudiante asigna a la información a que } \\
\text { tiene acceso, según la confianza que atribuye a la fuente que la recomienda. }\end{array}$ \\
\hline Pregunta $n^{0} 21$ & Reacción ante la información & $\begin{array}{l}\text { Valoración y respuesta crítica del estudiante frente a la información a que tiene } \\
\text { acceso. }\end{array}$ \\
\hline Pregunta $n^{0} 36$ & Herramientas para la reflexión & $\begin{array}{l}\text { Espacios que el estudiante utiliza para expresar sus pensamientos acerca de } \\
\qquad \text { los aprendizajes realizados. }\end{array}$ \\
\hline
\end{tabular}

Fuente: Elaboración propia.

\section{Análisis de datos}

En coherencia con los objetivos, el diseño de la investigación y la muestra participante, se ha realizado un análisis descriptivo de cada variable categórica, recogiendo en el siguiente apartado aquellos resultados (mostrados en porcentajes) de mayor puntuación. Además, y teniendo en cuenta la naturaleza de las variables utilizadas (todas son categóricas), se ha trabajado con ellas de manera simultánea realizando asociaciones que permitan establecer si hay relaciones entre las variables. Para ello, se ha descrito en primer lugar el comportamiento conjunto de aquellas variables (de dos en dos) que podrían aportar información relevante. Posteriormente, se ha averiguado si existe asociación y su grado de intensidad. Para la primera tarea se utilizan tablas de contingencia y para la segunda se hace uso de la prueba X2 de Pearson sobre independencia con el estadístico ji-cuadrado y la medida de asociación coeficiente de contingencia $C$, siendo el coeficiente de contingencia máximo 0.86 en todos los casos. Se han seleccionado aquellas relaciones entre variables que son estadísticamente significativas y muestran un grado de asociación alto (mayor del 75\%) y moderadamente alto (entre 60\% y 75\%) tras comparar en cada asociación el coeficiente de contingencia $C$ y coeficiente de contingencia máximo.

Este análisis permite identificar las estrategias y herramientas en red que los estudiantes universitarios participantes en el estudio utilizan para efectuar el tratamiento crítico de la información en su proceso de gestión del aprendizaje.

Para el análisis realizado se han reagrupado los valores de las respuestas utilizados en el cuestionario CAPPLE completo en tres categorías: valores relativos a opciones de respuesta positivos (totalmente de acuerdo y de acuerdo), valores relacionados con opciones de respuesta neutrales y valores sobre opciones de respuesta negativos (totalmente en desacuerdo y en desacuerdo. 
Para la realización del tratamiento y el análisis de los datos recogidos se utilizó el programa estadístico IBM SPSS (versión 22 para Windows).

\section{Resultados}

\section{Valoración de herramientas de aprendizaje}

Tres son los factores principales que determinan el número y la variedad de herramientas en red que los estudiantes utilizan para aprender (tabla 4): el primero es el tiempo que disponen para llevar a cabo un aprendizaje determinado (80.3\%) que, a su vez, se encuentra asociado con el segundo, la importancia que le dan a ese aprendizaje $(73.5 \%) X^{2}(9,2054)=1799.190 a, p<0.001, c=0.683$. La valoración académica o profesional que va a tener ese aprendizaje (67.5\%) es el tercer factor determinante que se encuentra asociado con el tiempo del que disponen para llevar a cabo cualquier aprendizaje $\mathrm{X}^{2}$ $(9,2054)=1251.353 a, p<0.001, c=0.615$. Sin embargo, el impacto que pueda tener en el prestigio del estudiante en red no influye en el número y la variedad de herramientas utilizadas para aprender, puesto que solamente el 20.9\% afirma lo contrario.

Tabla 4- El número y la variedad de herramientas en red que utilizan los estudiantes para aprender depende de...

\begin{tabular}{|c|c|c|c|c|c|}
\hline & & $\begin{array}{l}\text { Tiempo que tengo para } \\
\text { ese aprendizaje }\end{array}$ & $\begin{array}{l}\text { Importancia que doy a } \\
\text { un aprendizaje }\end{array}$ & $\begin{array}{c}\text { Valoración académica o } \\
\text { profesional que va a tener ese } \\
\text { aprendizaje }\end{array}$ & $\begin{array}{l}\text { Impacto que tendrá en mi } \\
\text { prestigio en la red }\end{array}$ \\
\hline $\mathrm{N}$ & $\begin{array}{c}\text { Válido } \\
\text { Perdidos }\end{array}$ & $\begin{array}{c}2054 \\
0\end{array}$ & $\begin{array}{c}2054 \\
0\end{array}$ & $\begin{array}{c}2054 \\
0\end{array}$ & $\begin{array}{c}2054 \\
0\end{array}$ \\
\hline Frecuencia & & $1650^{*}$ & $1509^{*}$ & $1386^{*}$ & $430^{*}$ \\
\hline Porcentaje & & $80.3 \%$ & $73.5 \%$ & $67.5 \%$ & $20.9 \%$ \\
\hline
\end{tabular}

* Opción de respuesta: siempre o a menudo.

Fuente: Elaboración propia.

\section{Credibilidad de la información: según origen}

Con los resultados obtenidos relacionados con la variable credibilidad de la información: según origen (tabla 5) se puede analizar el grado de veracidad que los estudiantes asignan a la información a la cual acceden según la fuente de procedencia. Dichos datos nos dicen que los estudiantes pocas veces o nunca cuestionan la información que reciben de expertos u otros profesionales del área (65.9\%) y de sus profesores (48.5\%), encontrando asociación entre ambos colectivos $X^{2}(9,2054)=1083.496^{\mathrm{a}}, \mathrm{p}<0.001, \mathrm{c}=0.588$. Sin embargo, el 45.8\% afirma que sí lo hace siempre o a menudo si la información que recibe procede de sus amigos y familiares.

Se encuentran datos muy similares si las fuentes de procedencia se encuentran mediadas por tecnologías, ya sean analógicas o digitales. Los estudiantes cuestionan 
la información que reciben siempre o a menudo de: redes sociales (72.1\%), medios de comunicación en red (62.5\%), foros (61.6\%), Blogs y páginas Web (61.2\%) y medios de comunicación tradicionales (57.6\%). Se encuentra una asociación alta y significativa entre los estudiantes que cuestionan la información que reciben de medios de comunicación tradicionales y aquellos que la reciben de medios de comunicación en red $X^{2}(9,2054)=1460.092^{a}, p<0.001, c=0.645$.

Tabla 5- Cuestiono la información que recibo de...

\begin{tabular}{|c|c|c|c|c|c|c|c|c|c|}
\hline & & $\begin{array}{l}\text { De expertos u otros } \\
\text { profesionales del área }\end{array}$ & $\begin{array}{c}\text { De mis } \\
\text { profesores }\end{array}$ & $\begin{array}{l}\text { De mis amigos } \\
\text { y familiares }\end{array}$ & $\begin{array}{l}\text { De Redes } \\
\text { Sociales }\end{array}$ & $\begin{array}{c}\text { Medios de } \\
\text { comunicación } \\
\text { en red }\end{array}$ & Foro & $\begin{array}{l}\text { Blog y } \\
\text { Web }\end{array}$ & $\begin{array}{c}\text { Medios de } \\
\text { comunicación } \\
\text { tradicionales }\end{array}$ \\
\hline \multirow{2}{*}{$\mathrm{N}$} & V & 2054 & 2054 & 2054 & 2054 & 2054 & 2054 & 2054 & 2054 \\
\hline & $P$ & 0 & 0 & 0 & 0 & 0 & 0 & 0 & 0 \\
\hline \multicolumn{2}{|c|}{ Frecuencia } & $1353^{\star \star}$ & $996^{\star \star}$ & $940^{*}$ & $1481^{*}$ & $1283^{*}$ & $1266^{*}$ & $1258^{*}$ & $1183^{*}$ \\
\hline \multicolumn{2}{|c|}{ Porcentaje } & 65.9 & 48.5 & 45.8 & 72.1 & 62.5 & 61.6 & 61.2 & 57.6 \\
\hline
\end{tabular}

* Opción de respuesta: siempre o a menudo. ** Opción de respuesta: pocas veces o nunca.

N: muestra. V: válido. P: perdidos.

Fuente: Elaboración propia.

\section{Credibilidad de la información: según recomendación}

Desde esta variable también se pretende analizar la credibilidad que los estudiantes otorgan a la información, pero en este caso según la confianza que atribuyen a la fuente que la recomienda. Cuatro son los ítems destacados de esta pregunta atendiendo a los resultados obtenidos.

Para el alumnado universitario encuestado, lo que más añade credibilidad a la información que reciben (tabla 6) es que la recomiende un experto (89.8\%) seguido de la posibilidad de que aparezca en varios recursos (artículos, libros, vídeos) en red (79.6\%). Por el contrario, el 50\% pocas veces o nunca añade credibilidad a la información recibida cuando es recomendada en las redes sociales. De otro lado, existe bastante dispersión en las respuestas cuando se pregunta a los estudiantes sobre la credibilidad añadida cuando la información se encuentra en una de las primeras posiciones de la búsqueda en Google (siempre o a menudo 31.1\%; a veces 28.4; pocas veces o nunca $37.5 \%$ ).

Tabla 6- Añade credibilidad a la información que recibo...

\begin{tabular}{|c|c|c|c|c|c|}
\hline & & $\begin{array}{l}\text { Que lo recomiende un } \\
\text { experto }\end{array}$ & $\begin{array}{c}\text { Que aparezca en } \\
\text { varios recursos }\end{array}$ & $\begin{array}{l}\text { Que sea recomendada en } \\
\text { redes sociales }\end{array}$ & $\begin{array}{l}\text { Que sea una de las primeras } \\
\text { posiciones de la búsqueda de Google }\end{array}$ \\
\hline $\mathrm{N}$ & $\begin{array}{l}\text { V } \\
\mathrm{P}\end{array}$ & $\begin{array}{c}2054 \\
0\end{array}$ & $\begin{array}{c}2054 \\
0\end{array}$ & $\begin{array}{c}2054 \\
0\end{array}$ & $\begin{array}{c}2054 \\
0\end{array}$ \\
\hline \multicolumn{2}{|c|}{ Frecuencia } & $1844^{*}$ & $1636^{*}$ & $1027^{\star \star}$ & $771^{\star *}$ \\
\hline \multicolumn{2}{|c|}{ Porcentaje } & $89.8 \%$ & $79.6 \%$ & $50 \%$ & $37.5 \%$ \\
\hline
\end{tabular}

* Opción de respuesta: siempre o a menudo. ** Opción de respuesta: pocas veces o nunca.

N: muestra. V: válido. P: perdidos.

Fuente: Elaboración propia. 


\section{Reacción ante la información}

En relación a la capacidad de valoración y respuesta crítica del estudiante frente a la información recibida (tabla 7), se encuentra que: el alumnado universitario encuestado siempre o a menudo la interpreta de manera reflexiva en base a argumentos que le ayudan a comprenderla (79.2\%); es consciente de que la interpreta según sus propios puntos de vista (78.9\%); la contrasta (67\%); valora la opinión dada por el grupo de usuarios (58.6\%); y piensa que no es veraz (55\%). Estos datos quedan confirmados cuando los estudiantes afirman que pocas veces o nunca (70.9\%) la información no debe cuestionarse.

Tabla 7- Ante la información que recibo...

\begin{tabular}{|c|c|c|c|c|c|c|c|}
\hline & & $\begin{array}{l}\text { La interpreto de manera } \\
\text { reflexiva en base a } \\
\text { argumentos que me } \\
\text { ayuden a comprenderla }\end{array}$ & $\begin{array}{l}\text { Soy consciente de } \\
\text { que la interpreto } \\
\text { según mis propios } \\
\text { puntos de vista }\end{array}$ & La contrasto & $\begin{array}{l}\text { Valoro la } \\
\text { opinión dada } \\
\text { por el grupo de } \\
\text { usuarios }\end{array}$ & $\begin{array}{l}\text { Pienso que no } \\
\text { siempre es veraz } \\
\text { o se corresponde } \\
\text { con la realidad }\end{array}$ & $\begin{array}{l}\text { Considero que no } \\
\text { debo cuestionarla, } \\
\text { sea cual sea su } \\
\text { origen }\end{array}$ \\
\hline $\mathrm{N}$ & $\begin{array}{l}\mathrm{V} \\
\mathrm{P}\end{array}$ & $\begin{array}{c}2054 \\
0 \\
\end{array}$ & $\begin{array}{c}2054 \\
0 \\
\end{array}$ & $\begin{array}{c}2054 \\
0 \\
\end{array}$ & $\begin{array}{c}2054 \\
0\end{array}$ & $\begin{array}{c}2054 \\
0\end{array}$ & $\begin{array}{c}2054 \\
0\end{array}$ \\
\hline \multicolumn{2}{|c|}{ Frecuencia } & $1627^{*}$ & $1620^{*}$ & $1387^{*}$ & $1203^{*}$ & $1130^{*}$ & $1456^{\star \star}$ \\
\hline \multicolumn{2}{|c|}{ Porcentaje } & $79.2 \%$ & $78.9 \%$ & $67.5 \%$ & $58.6 \%$ & $55 \%$ & 70.9 \\
\hline
\end{tabular}

* Opción de respuesta: siempre o a menudo. ** Opción de respuesta: pocas veces o nunca.

$\mathrm{N}$ : muestra. V: válido. P: perdidos.

Fuente: Elaboración propia.

\section{Herramientas para la reflexión}

Cuatro fueron los ítems utilizados en el cuestionario para tratar de determinar los espacios (blog personal, documento de Word, calendario electrónico, hoja de papel o diario) que el estudiante utiliza para expresar sus pensamientos acerca de los aprendizajes realizados. Sin embargo, los resultados indican que solamente existen datos significativos en dos ítems (tabla 8).

Por un lado, los estudiantes no usan (40\%) o pocas veces o nunca (44\%) el blog personal como herramienta para reflejar la reflexión de lo que van aprendiendo. De otro lado, se encuentran similares resultados en relación al uso del calendario electrónico para reflexionar sobre los aprendizajes (pocas veces o nunca $47.8 \%$ y no usa $40.8 \%)$, encontrando una asociación significativa y alta entre ambas variables $X^{2}(9$, 2054) $=1234.192^{\mathrm{a}}, \mathrm{p}<0.001, \mathrm{c}=0.613$.

Tabla 8- Suelo reflejar la reflexión sobre lo que voy aprendiendo...

\begin{tabular}{ccccccc}
\hline & $\vdots$ & & $\vdots$ & En un blog personal & $\vdots$ & En un calendario electrónico \\
\hline \multirow{2}{*}{$\mathbf{N}$} & $\vdots$ & Válido & 2054 & $\vdots$ & 2054 \\
& & Perdidos & 0 & $\vdots$ & 0 \\
\hline Frecuencia & $\vdots$ & & $\vdots$ & $903^{\star \star}$ & $\vdots$ & $982^{\star \star}$ \\
\hline Porcentaje & $\vdots$ & & $\vdots$ & $44 \%$ & $\vdots$ & $47.8 \%$ \\
\hline
\end{tabular}

** Opción de respuesta: pocas veces o nunca.

$\mathrm{N}$ : muestra.

Fuente: Elaboración propia. 


\section{Conclusión y discusión}

Diversos estudios (OSSA-CORNEJO et al., 2018; MADARIAGA; SCHAFFERNICHT, 2013; ZELEITA; ORTIZ, 2018) corroboran que el pensamiento crítico es una habilidad necesaria para el desarrollo profesional. Además, consideran determinante que las instituciones de educación superior establezcan programas y estrategias que faciliten el desarrollo de la capacidad reflexiva, que a su vez son valoradas positivamente por los estudiantes para su desempeño profesional. Desde este enfoque, los resultados de esta investigación contribuyen a identificar las estrategias y las herramientas en red utilizadas de forma habitual por estudiantes universitarios españoles de último curso para efectuar un tratamiento crítico de la información y gestionar su proceso de aprendizaje. Partiendo de los datos analizados, considerando la limitación derivada de una muestra amplia aunque no representativa de universitarios españoles $\mathrm{y}$, a pesar de las dificultades que conlleva evaluar la capacidad crítica de los estudiantes (DANIEL et al., 2003), podemos afirmar que la mayoría de los estudiantes de las universidades participantes en el estudio son conscientes de la necesidad de contrastar y aplicar un pensamiento crítico sobre las informaciones que reciben durante su proceso formativo.

Frente a la información recibida, los estudiantes en general aseguran contrastarla e interpretarla de manera reflexiva, aportando argumentos que les ayudan a comprenderla. Más aún, dicen ser conscientes de que estas interpretaciones las realizan desde sus propios puntos de vista.

Aunque la gran mayoría considera necesario interpretar de modo reflexivo la información recibida, en gran medida, se cuestionan que no siempre dicha información es verídica o se corresponde con la realidad. Hay que tener en cuenta que una minoría de estudiantes afirma que no considera necesario cuestionarse la información sea cual sea su origen, hecho que sorprende al tratarse de alumnos que están a punto de terminar sus estudios universitarios y que deberían de haber desarrollado estrategias de reflexión y de pensamiento crítico al final sus estudios de superiores. Lo cual nos indica que nuestros estudiantes universitarios siguen teniendo deficiencias y dificultades para desplegar el pensamiento crítico en el aula (HORN, 2000).

En cuanto a la credibilidad de la información, el posicionamiento crítico varía sustancialmente según el origen de la fuente de información. Existen relaciones significativas entre los estudiantes (la gran mayoría) que consideran fiable y de gran credibilidad las informaciones dadas por expertos y profesionales del campo de estudio y entre aquellos que lo hacen cuando la información procede de los profesores que les imparten las asignaturas. En cambio, no dan tanta credibilidad si las informaciones tienen su origen en fuentes externas al contexto académico, ya sean del entorno personal más próximo (amigos y familiares), provenga de los medios de comunicación (tradicionales o en red), la reciban a través de aplicaciones y espacios en red (blogs y páginas web, redes sociales, foros, etc.), ni tampoco si se trata de informaciones que se han encontrado en las primeras posiciones de un motor de búsqueda genérico. Se podría decir que esta conciencia crítica los lleva a cuestionar de forma generalizada las informaciones recibidas fuera del ámbito académico, especialmente si proviene de redes sociales como pueden ser 
informaciones virales o que sean trendings topic en twitter. Esta realidad nos indica que los estudiantes universitarios son más tradicionales como aprendices de lo que podríamos pensar (PRENDES et al., 2016). Además, esta falta de confianza en la información que le llega desde entornos informales puede deberse: a) a una falta de capacidad reflexiva que le permita establecer criterios de búsqueda, acceso y selección de información, sea en el entorno que sea, o bien; b) a que no haya alcanzado un nivel de competencia razonable en la selección crítica de la información. En este sentido, varios estudios (EGAÑA; BIDEGAIN; ZUBEROGOITIA, 2013; FUENTES; MONEREO, 2008; GUTIÉRREZ et al., 2017; GUTIÉRREZ; SERRANO, 2018) concluyen que los estudiantes universitarios deben mejorar su competencia digital en el área de acceso a la información, en la que indudablemente la capacidad crítica tiene un espacio destacado.

Retomando los datos analizados, los estudiantes valoran el uso de diferentes herramientas de aprendizaje, principalmente en función del tiempo que disponen para invertir en el desarrollo de un proceso de aprendizaje concreto. El tiempo disponible está relacionado de manera significativa tanto con la valoración académica o profesional que va a tener ese aprendizaje como con la importancia que los estudiantes le dan a ese aprendizaje. De otro lado, los estudiantes no suelen utilizar herramientas para reflexionar, encontrando relaciones significativas entre los que no hacen uso del blog personal y entre los que tampoco utilizan el calendario electrónico para reflejar sus reflexiones.

En cambio, no se plantean, ni muestran interés en el posible impacto que pueda tener su proceso y conocimiento adquiridos en su prestigio en la red. Frente a la elevada presencia que los jóvenes universitarios tienen en la red, este resultado puede interpretarse como un indicio de la separación que, de facto, hacen entre su actividad formativa y su presencia en la red, que posiblemente esté más vinculada al ocio (SÁNCHEZ; SERRANO; PRENDES, 2013). Cuando, precisamente, los PLE se consideran como un espacio de confluencia y conexión entre lo formal, lo no formal y lo informal.

Volviendo a la conclusión general que apuntábamos más arriba cabe pensar que el pensamiento crítico puede llegar a ser transferido a otros contextos, como son el personal y el profesional. De confirmarse este planteamiento hipotético -que obviamente requiere de estudios ad hoc- vendría a corroborar que, en la gestión del proceso de aprendizaje, el pensamiento crítico es una de las variables que contribuye al desarrollo del PLE como espacio de confluencia de los aprendizajes desplegados por las personas en todo tipo de contextos y circunstancias.

Por otra parte, entre los temas pendientes que abre este trabajo se destaca con nitidez la profundización teórica y práctica sobre la capacidad predictiva de los PLE: a) como herramienta de evaluación y de aprendizaje en futuras acciones de desempeño laboral y; b) en cómo mejora este desempeño tras una sólida formación en el tratamiento crítico de la información desplegada durante los estudios universitarios de grado. También queda pendiente abordar, como ya lo han hecho Chaves-Barboza y otros autores (2017), cuál es papel de la ayuda docente y del tipo de herramientas digitales en los procesos de autorregulación a través de los PLE.

En esta relación entre los PLE, el desempeño profesional y el tratamiento crítico de la información orientado a la gestión del propio aprendizaje, además de proseguir con la 
investigación, el profesorado universitario debe contemplar estrategias que permitan un desarrollo sólido de esta competencia y faciliten su transferencia al entorno laboral. La adopción de los PLE en la formación universitaria:

[...] requiere un esfuerzo por visibilizar, analizar, reflexionar, construir un estilo y ser conscientes de hacia dónde se quiere ir. La persona que elabora su PLE tiene que tomar decisiones sobre qué elementos poner y cómo se relacionan los elementos. Ello le exige, primero, pensar sobre qué hace para aprender, qué le gusta o qué siente como más propio, como estrategia de aprender. El segundo paso, que se apoya en el primero, será identificar el papel que desempeña para la determinación de su propio proceso de aprendizaje. Y, por último, habrá que autorregular su proceso de aprender trazando un camino personal y reformulando su PLE en ese proceso, ya que es necesario que sea repensado y enriquecido para que así refleje el proceso continuo de aprender en cada etapa de la persona. (CEBREIRO, 2017, p. 145).

Complementando las presentadas en Gutiérrez y otros autores (2017), apuntamos a continuación algunas de estas posibles líneas de actuación que pueden adoptarse tanto en las aulas universitarias como en los espacios de práctica profesional o en el uso personal que cada estudiante hace de su propio PLE.

Las estrategias prioritarias deben apuntar inicialmente a la sensibilización de los estudiantes, y parte del profesorado, respecto a la necesidad de habituarse a ser reflexivo y crítico con la información que maneja evitando convertirse en un consumidor pasivo, acrítico y conformista. Sin una motivación personal auténtica, resulta difícil, seguramente imposible, adoptar una actitud proactiva que derive en prácticas sistemáticas de análisis crítico y reflexivo de la información. La identificación de necesidades de información, la planificación de las búsquedas y la adopción de criterios estrictos de indagación, el acceso selectivo a las fuentes, la definición y aplicación de criterios de selección en función de las necesidades que motivan el acceso a la información, el contraste y triangulación de los contenidos localizados y la lectura profunda y reflexiva de dichos contenidos deberían estar siempre presentes en las actividades formativas y aplicarse de forma rutinaria en el entorno laboral. Incluso pueden adoptarse procedimientos de trabajo propios de los curadores de contenidos (ROMERO, 2016; LEE, 2016) de modo que se promueva el logro de un nivel de competencia superior que ahonde en el desarrollo del pensamiento crítico. En cualquier caso, resulta imprescindible promover que los estudiantes universitarios deban, en aquellas actividades que requieren de tratamiento crítico de la información, emitir siempre juicios propios, pues compartimos con Lipman (1991) que los juicios son los productos del pensamiento crítico.

\section{Referencias}

ALA-MUTKA, Kirsti; PUNIE, Yves; REDECKER, Christine. Digital competence for lifelong learning. Sevilla: Institute for Prospective Technological Studies: JRC, 2008. Disponible en: <ftp://ftp.jrc.es/pub/EURdoc/ EURdoc/JRC48708.TN.pdf>. Acceso en: 12 en. 2018. 
ANDERSON, Peter. Assessment and development of Executive Function (EF) during chilhood. Child Neuropsychology, Orlando, v. 8, n. 2, p. 71-82, 2002. Disponible en: <https://doi.org/10.1076/ chin.8.2.71.8724>. Acceso en: 6 febr. 2018

ATO, Manuel; LÓPEZ, Juan José; BENAVENTE, Ana. Un sistema de clasificación de los diseños de investigación en psicología. Anales de Psicología, Murcia, v. 29, n. 3, p. 1038-1059, 2013. Disponible en: $<$ http://dx.doi.org/10.6018/analesps.29.3.178511>. Acceso en: 15 dic. 2017.

BENKLER, Yochai. Innovación distribuida y creatividad, trabajo colaborativo y el procomún en una economía en red. Madrid: Open Mind: BBVA, 2014. Disponible en: <https://www.bbvaopenmind.com/ articulo/innovacion-distribuida-y-creatividad-trabajo-colaborativo-y-el-procomun-en-una-economia-enred/?fullscreen=true $>$. Acceso en: 15 nov. 2017.

BURBULES, Nicholas. El aprendizaje ubicuo: nuevos contextos, nuevos procesos. Entramados, Mar del Plata, v. 1, n. 1, p. 131-134, 2014.

BUTLER, Heather et al. Extending the validity of halpern critical thinking assessments: cross-national applications. Thinking Skills and Creativity, Cambridge, v. 7, n. 2, p. 112-121, Aug. 2012.

CARRETERO, Stephanie; VUORIKARI, Riina; PUNIE, Yves. DigComp 2.1: the digital competence framework for citizens with eight proficiency levels and examples of use. Seville: Joint Research Centre, 2017. Disponible en: <http://dx.doi.org/10.2760/38842>. Acceso en: 10 jun. 2017.

CASTAÑEDA, Linda; ADELL, Jordi. El desarrollo profesional de los docentes en entornos personales de aprendizaje (PLEs). In: ROIG VILA, Rosabel (Ed.). La práctica educativa en la sociedad de la información: innovación a través de la investigación. Alcoy: Marfil, 2011. p. 83-96. Disponible en: <http://digitum. um.es/xmlui/bitstream/10201/24647/1/CastanedaAdell2011 preprint.pdf>. Acceso en: 20 sept. 2017.

CASTAÑEDA, Linda; ADELL, Jordi. Entornos personales de aprendizaje: claves para el ecosistema educativo en la red. Alcoy: Marfil, 2013.

CASTELLS, Manuel. Comunicación y poder. Madrid: Alianza, 2009.

CEBREIRO, Beatriz. EI PLE, un elemento importante en el camino hacia aprender a aprender. In: PRENDES ESPINOSA, María Paz; ROMÁN GARCIA, Marimar (Coord.). Entornos personales de aprendizaje: una visión actual de cómo aprender con tecnologías. Barcelona: Octaedro, 2017. p. 145-149.

CHAVES-BARBOZA, Eduardo et al. Actions and achievements of self-regulated learning in personal environments: research on students participating in the graduate program in preschool education at the University of Granada. Journal of New Approaches in Educational Research, Alicante, v. 6, n. 2, p. 135-143, 2017. Disponible en: <http://dx.doi.org/10.7821/naer.2017.7.236>. Acceso en: 20 dic. 2017.

CONOLE, Gráinne et al. 'Disruptive technologies', 'pedagogical innovation': what's new? Findings from an in-depth study of students' use and perception of technology". Computers \& Education, Washington, DC, v. 50, n. 2, p. 511-524, 2008. 
CONOLE, Gráinne. Las pedagogías de los entornos personales de aprendizaje. In: CASTAÑEDA, Linda; ADELL, Jordi (Ed.). Entornos personales de aprendizaje: claves para el ecosistema educativo en red. Alcoy: Marfil, 2013. p. 185-188.

DANIEL, Marie-France et al. ¿Qué es el pensamiento dialógico crítico? Perfiles Educativos, Ciudad de México, v. 25, n. 102, p. 22-39, 2003. Disponible en: <http://www.iisue.unam.mx/perfiles/articulo/2003102-que-es-el-pensamiento-dialogico-critico.pdf>. Acceso en: 8 en. 2018.

DEZUANNI, Michael; MONROY, Andrés. Prosumidores interculturales: la creación de medios digitales globales entre los jóvenes. Comunicar, Huelva, n. 38, p. 59-66, 2012. Disponible en: <https://doi. org/10.3916/C38-2012-02-06>. Acceso en: 10 en. 2018.

DÍAZ BARRIGA, Frida. Habilidades de pensamiento crítico sobre contenidos históricos en alumnos de bachillerato. Revista Mexicana de Investigación Educativa, Ciudad de México, v. 6, n. 13, p. 1-20, 2001. Disponible en: <http://www.redalyc.org/pdf/140/14001308.pdf>. Acceso en: 20 en. 2018.

DWYER, Christopher et al. An integrated critical thinking framework for the 21 st century. Thinking Skills and Creativity, Cambridge, v. 12, p. 43-52, 2014. Disponible en: <http://www.sciencedirect.com/science/ article/pii/S1871187114000030 >. Acceso en: 5 en. 2018.

EGAÑA, Txema; BIDEGAIN, Eneko; ZUBEROGOITIA, Aitor. ¿Cómo buscan información académica en internet los estudiantes universitarios? Lo que dicen los estudiantes y sus profesores. Edutec, n. 43, p. 1-15, 2013. DOl: http://dx.doi.org/10.21556/edutec.2013.43.332

FERRARI, Anusca. DIGCOMP: a framework for developing and understanding digital competence in Europe. Sevilla: JRC: Institute for Prospective Technological Studies, 2013. Disponible en: <http://omk-obrazovanje. gov.rs/wp-content/uploads/2015/02/A-Framework-for-Digital-Competence-in-Europe.pdf>. Acceso en: 8 en. 2018.

FUENTES, Marta; MONEREO, Carlos. Cómo buscan información en internet los adolescentes. Revista Investigación en la Escuela, Sevilla, n. 64, p. 45-58, 2008. Disponible en: <http://www. investigacionenlaescuela.es/articulos/64/R64_4.pdf>. Acceso en: 20 dic. 2017.

GALLARD0, Eliana Esther. An investigation of the social and academic uses of digital technology by university students. Tarragona: Universitat Rovira i Virgili, 2015. 218 p. Tesis (Doctorado en Tecnología Educativa) Facultat de Ciènces de l'Educació, Universitat Rovira i Virgili, Tarragona, 2015. Disponible en: <http://www. tesisenred.net/handle/10803/277423>. Acceso en: 7 febr. 2018.

GISBERT, Mercè; ESTEVE, Francesc. Digital learners: la competencia digital de los estudiantes universitarios. La Cuestión Universitaria, n. 7, p. 48-59, 2011. Disponible en: <https://www.researchgate. net/profile/Francesc_Esteve/publication/221680100_Digital_Learners_la_competencia_digital_de_los_ estudiantes_universitarios/links/09e4150b33eb28580f000000.pdf>. Acceso en:

GROS, Begoña. La caída de los muros del conocimiento en la sociedad digital y las pedagogías emergentes. Teoría de la Educación. Educación y Cultura en la Sociedad de la Información, Salamanca, v. 16, n 1, p. 58-68, 2015. Disponible en: <http://dx.doi.org/10.14201/eks20151615868>. Acceso en: 16 oct. 2017. 
GUALLAR, Javier; LEIVA-AGUILERA, Javier. El content curator: guía básica para el nuevo profesional de internet. Barcelona: UOC, 2013.

GUTIÉRREZ, Isabel et al. Estrategias y herramientas para aprender: descripción de los PLE de los estudiantes universitarios españoles y propuestas para la mejora de la respuesta institucional. In: PRENDES, María Paz; ROMÁN, María del Mar. Entornos personales de aprendizaje: una visión actual de cómo aprender con tecnologías. Barcelona: Octaedro, 2017. p. 65-88.

GUTIÉRREZ, Juan Antonio; SERRANO, José Luis. Análisis de los procesos de búsqueda, acceso y selección de información digital en futuros maestros. Digital Education Review, Barcelona, n. 34, p. 76-90, 2018.

HALPERN, Diane; NUMMEDAL, Susan (Ed.) Psychologist teach critical thinking. Teaching of Psychology, Washington, DC, v. 22, n. 1, p. 4-83, febr. 1995.

HORN, Raymond. Becoming a critical teacher. In: WEIL, Danny; ANDERSON, Holly Kethleen (Ed.). Perspectives in critical thinking: essays by teachers in theory and practice. New York: Peter Lang, 2000. p. 139-172. (Counterpoints; v. 110).

JISC - Joint Information Systems Committee. Learning in a digital age: extending higher education opportunities for lifelong learning. Bristol: Hefce, 2012. Disponible en: <http://www.jisc.ac.uk/whatwedo/ programmes/elearning/digilifelong.aspx>. Acceso en: 10 febr. 2018.

KERHOHAN, David. Education is broken, somebody should do something. Followers of the Apocalypse, 2013. Disponible en: <http://followersoftheapocalyp.se/education_is_broken/\#sthash.ZsbMw5Jh.dpuf>. Acceso en: 10 nov. 2017.

KOP, Rita. The Challenges to connectivist learning on open online networks: learning experiences during a massive open online course. Irrodl, Athabasca, v. 12, n. 3, 2011. Disponible en: <http://dx.doi.org/10.19173/ irrodl.v12i3.882>. Acceso en: 10 febr. 2018.

LEE, Kevan. How to curate content: the secret sauce to getting noticed, becoming an influencer, and having fun onlin. Business 2 Community, 2016. Disponible en: <https://www.business2community.com/brandviews/ buffer/curate-content-secret-sauce-getting-noticed-becoming-influencer-fun-online-01650626>. Acceso en: 29 dic. 2018.

LIPMAN, Matthew. Thinking in education. Cambridge: Cambridge University Press, 1991.

LOVELESS, Avril; WILLIAMSON, Bean. Nuevas indentidades de aprendizaje en la era digital: creatividad, educación, tecnología, sociedad. Madrid: Narcea, 2017.

MADARIAGA, Patricio; SCHAFFERNICHT, Martin. Uso de objetos de aprendizaje para el desarrollo del pensamiento crítico. Revista de Ciencias Sociales, San José, v. 19, n. 3, p. 472-484, 2013.

MARTíN, Eduardo et al. Estructura factorial y fiabilidad de un cuestionario de estrategias de aprendizaje en universitarios: CEA-U. Anales de Psicología, Murcia, v. 23, n. 1, p. 1-6, 2007. Disponible en: <http://www. redalyc.org/articulo.oa?id=16723101>. Acceso en: 10 febr. 2018. 
MAYER, Richard; GOODCHILD, Fiona. The critical thinker. Santa Barbara: University of California: Wm. C. Brown Publishers, 1990.

MCMILLAN, James. Enhacing college students' critical thinking: a review of studies. Research in Higher Education, Georgia, v. 26, n. 1, p. 3-29, 1987.

MIDGLEY, Carol et al. Manual for the paterrns off adaptive learrning scales. Michigan: University of Michigan, 2000. Disponible en: <http://www.umich.edu/ pals/PALS\%202000_V13Word97.pdf>. Acceso en: 10 jun. 2016.

MONROY, Miguel. El pensamiento didáctico del profesor: un estudio con profesores de ciencias históricosociales del Colegio de Bachilleres y del Colegio de Ciencias y Humanidades. Ciudad de México: UNAM, 1998. 156 p. Tesina (Maestría en Psicología Educativa) - Facultad de Psicología, Universidad Autónoma de México, Ciudad de México, 1998.

NAVARRETE-CAZALES, Zaira. ¿Otra vez la identidad? Un concepto necesario pero imposible. Revista Mexicana de Investigación Educativa, Ciudad de México, v. 20, n. 65, p. 461-479, 2015. Disponible en: $<$ http://www.redalyc.org/articulo.oa?id=14035408007>. Acceso en: 6 en. 2018.

NIETO, Ana María, SAIZ, Carlos. Skills and dispositions of critical thinking: are they sufficient? Anales de Psicología, Murcia, v. 27, n. 1, p. 202-209, 2011. Disponible en: <http://www.redalyc.org/articulo. oa?id=16717018024>. Acceso en: 5 dic. 2017.

OSSA-CORNEJO, Carlos et al. Evaluación del pensamiento crítico y científico en estudiantes de pedagogía de una universidad chilena. Revista Electrónica Educare, Heredia, v. 22, n. 2, p. 1-18, 2018. Disponible en: $<$ http://dx.doi.org/10.15359/ree.22-2.12>. Acceso en: 20 nov. 2017.

PACHECO, Diego. A professional service-learning program and its impact on critical thinking skills and professional responsibility development in higher education students: a case study. Ohio: [s. n.], 2001. Research proposal, doctoral program, College in Education Yucatan Autonomous University and Ohio University.

PARD0, Antonio et al. Análisis de datos en ciencias sociales y de la salud I. Madrid: Síntesis, 2015.

PEÑA LÓPEZ, Ismael. ¿Con qué aprender en red? Estrategias y herramientas para la apertura y disrupción de las instituciones educativa. In: GROS, Begoña; SUÁREZ GUERRERO, Cristóbal. Pedagogía red: una educación para tiempos de internet. Barcelona: Octaedro, 2016. p. 77-104.

PINTRICH, Paul et al. A manual for the use of the Motivated Strategies of Learning Questionnaire (MSLQ). Washington, DC.: National Center for Research to Improve Postsecondary Teaching and Learning, Office of Educational Research and Improvement, 1991. Disponible en: <http://files.eric.ed.gov/fulltext/ED338122. pdf>. Acceso en: 7 en. 2017.

PRENDES, María Paz. Competencias TIC para la docencia en la Universidad Pública Española: indicadores y propuestas para la definición de buenas prácticas: Programa de Estudio y Análisis. Informe del Proyecto EA2009-0133. Murcia: Secretaría del Estado de Universidades e Investigación, 2010. Disponible en: <http://www.um.es/competenciastic>. Acceso en: 10 my. 2017. 
PRENDES, María Paz. CAPPLE: explorando los PLE de los futuros profesionales. In: CASTAÑEDA, Linda; ADELL, Jordi (Ed.). Entornos personales de aprendizaje: claves para el ecosistema educativo en red. Alcoy: Marfil, 2013. p. 173-175.

PRENDES, María Paz; GUTIÉRREZ, Isabel. Competencias tecnológicas del profesorado en las universidades españolas. Revista de Educación, Madrid, n. 361, p. 196-222, 2013. Disponible en: <http://dide.minedu. gob.pe/xmlui/handle/123456789/2432>. Acceso en: 14 dic. 2017.

PRENDES, María Paz et al. Componentes básicos para el análisis de los PLE de los futuros profesionales españoles: en los albores del Proyecto CAPPLE. Edutec, Palma de Mallorca, v. 47, p. 1-15, 2014. Disponible en <http://edutec.rediris.es/Revelec2/Revelec47/n47_Prendes-Castaneda-Ovelar-Carrera.html>. Acceso en: 16 jun. 2017.

PRENDES, María Paz et al. Validation of a questionnaire on work and learning habits for future professionals: exploring personal learning environments. Relieve, Valencia, v. 22, n. 2, p. 1-45, 2016. Disponible en: <http://dx.doi.org/10.7203/relieve.22.2.7228>. Acceso en: 10 nov. 2017.

PRENSKY, Marc. Digital natives, digital immigrants. On the Horizon, Bingley, v. 9, n. 5, p. 1-6, 2001. Disponible en: <http://dx.doi.org/10.1108/10748120110424816>. Acceso en: 8 oct. 2017.

ROMERO, María José Iniciación a la curación de contenidos en la universidad: una experiencia en el área de psicobiología. RED - Revista de Educación a Distancia, Murcia, n. 49, p. 1-32, abr. 2016. Disponible en: <http://dx.doi.org/10.6018/red/49/6>. Acceso en: 20 nov. 2017.

SÁNCHEZ, María del Mar; SERRANO, José Luis; PRENDES, María Paz. Análisis comparativo de las interacciones presenciales y virtuales de los estudiantes de enseñanza secundaria obligatoria. Educación XXI, Madrid, v. 16, n. 1, p. 351-374, 2013. doi: https://doi.org/10.5944/educxx1.16.1.730

SUÁREZ-GUERRERO, Cristóbal. Entorno y comunicación en el e-learning. In: GROS SALVAT, Begoña; MAS, Xavier (Ed.). La comunicación en los espacios virtuales: enfoques y experiencias de formación en línea. Barcelona: UOC, 2013. p. 21-50.

VUORIKARI, Riina et al. DigComp 2.0: the digital competence framework for citizens. Update phase 1: the conceptual reference model (No. JRC101254). Luxembourg: Office of the European Union, 2016. EUR 27948 EN. doi:10.2791/11517

WHITE, David; LE CORNU, Alison. Visitors and residents: a new typology for online engagement. First Monday, Chicago, v. 16, n. 9, 2011. Disponible en: <http://firstmonday.org/ojs/index.php/fm/article/ viewArticle/3171>. Acceso en: 1 febr. 2018.

WILSON, Brent. Metaphors for instruction: why we talk about learning environments. Educational Technology, New York, v. 35, n. 5, p. 25-30, 1995.

ZELEIETA, Edu; ORTIZ, Igo Camino. El desarrollo del pensamiento crítico en la formación inicial del profesorado: análisis de una estrategia pedagógica desde la visión del alumnado. Profesorado, Granada, v. 22, n. 1, p. 197-214, 2018. 
Recibido en: 23.03.2018

Revisiones en: 19.06 .2018 Aprobado en: 21.08.2018

José Luis Serrano Sánchez es doctor y profesor de Tecnología Educativa en la Universidad de Murcia (España). Miembro del grupo de investigación de Tecnología Educativa (GITE) de la Universidad de Murcia. Director ejecutivo de Revista Interuniversitaria de Investigación en Tecnología Educativa (RIITE). Web profesional: www.jlserranosanchez.es

Xavier Carrera es doctor en Psicopedagogía y Premio Extraordinario de Doctorado. Profesor titular en el Departamento de Pedagogía y Psicología de la Universidad de Lleida (España). Coordinador del grupo de investigación consolidado 2017 SGR 1700 (COMPETECS Competencias, Tecnología y Educación en Sociedad). Coordinador en la Universidad de Lleida del Programa de Doctorado Interuniversitario en Tecnología Educativa.

Enric Brescó Baiges es doctor en Tecnología Educativa y licenciado en Comunicación Audiovisual y Psicopedagogía. Profesor de la Facultad de Educación, Psicología y Trabajo Social de la Universidad de Lleida. Miembro del grupo de investigación consolidado (SGR 1311) Competencias, Tecnología, Educación y Sociedad (COMPETECS). Especialista en elearning en la Unidad de Soporte y Atención a la Actividad Docente.

Cristóbal Suárez-Guerrero es doctor en Educación en formación en espacios virtuales por la Universidad de Salamanca y profesor del Departamento de Didáctica y Organización Escolar de la Universitat de València. Líneas de investigación: pedagogía digital, política y cultura educativa digital y cooperación en red. 